\title{
Are Selected Bone Marrow Stem Cells More Effective than Unselected Ones in Patients with Chronic Myocardial Infarction?
}

\section{Hyunsuk Jeong ${ }^{1}$, Hyeon Woo Yim ${ }^{1 *}$, Hun-Jun Park ${ }^{2}$, Sona Jeong ${ }^{3}$ and Hyun-bin Kim ${ }^{4}$}

${ }^{1}$ Department of Preventive Medicine, the Catholic University of Korea, Republic of Korea

${ }^{2}$ Division of Cardiovascular Medicine, Seoul St. Mary's Hospital, Republic of Korea

${ }^{3}$ Medical Library, Seoul St. Mary's Hospital, Republic of Korea

${ }^{4}$ Clinical Research Coordinating Center, Catholic Medical Center, Republic of Korea

\begin{abstract}
Introduction: The aim of the study was to examine the effectiveness of selected bone marrow-derived stem cell (BMSC) on improving Left Ventricular Ejection Fraction (LVEF) from Randomized Controlled Trials (RCTs) to treat patients with Chronic Myocardial Infarction (CMI).

Methods: We searched Medline from 1946 to March 2012 for studies of BMSC transplantation in patients with CMI. The included studies met the following criteria: RCTs, CMI patients who received Coronary Artery Bypass Graft $(\mathrm{CABG}), \mathrm{BMSC}$ were infused intramuscularly, cell injection in peri-infarct zone, and studies that had up to 6 month follow-up.

Results: The initial search identified 8,433 references, of which 7 RCTs met the inclusion criteria. Selected bone marrow stem cells were injected in three of the 7 trials while unselected BMSC was injected to the treatment group in the rest 4 trials. The treatment effects of the studies in which the treatment group was injected with CD34+ and CD133+ were greater than the studies that used unselected BMSC (7.66\%, 95\% Cl: 4.16-11.15 vs. 4.77\%; 95\% $\mathrm{Cl}$ : 2.08-7.46). Planned sub-group analyses revealed that the treatment effects on improvement in LVEF differed according to the measurement tools used on outcome assessment, treatment blindness, and methods of surgery.

Conclusion: Selected BMSC appeared to show more effective than unselected BMSC. However, the intervention effect of selected BMSC might be overestimated because the studies tended to use less rigorous designs, less precise outcome measures, and different methods of surgery than those using unselected BMSC. Therefore these treatment effects of selected BMSC should be interpreted cautiously.
\end{abstract}

Keywords: Bone marrow stem cell; Chronic myocardial infarction; Systematic review

Abbreviations: BMSC: Bone Marrow Stem Cell; CMI: Chronic Myocardial Infarction; CABG: Coronary Artery Bypass Graft; LVEF: Left Ventricular Ejection Fraction; MRI: Magnetic Resonance Imaging; SPECT: Single Photon Emission Computed Tomography; OPCAB: Off-pump Coronary Artery Bypass Grafting; RCTs: Randomized Controlled Trials

\section{Introduction}

Studies have shown global and regional functional improvements after Bone Marrow Stem Cell (BMSC) have been injected into viable, peri-infarct areas of chronically ischemic myocardium [1-3]. Bone marrow-derived CD34+ and CD 133+ cells, which exhibit endothelial phenotypes, have been shown to contribute to neovascularization. Also, intramyocardial injection of purified CD133+ BMSC that was involved in their direct application into the diseased myocardium at the time of Coronary Artery Bypass Graft (CABG). Bone marrow or mobilized peripheral blood progenitor cells might play a role in the revascularization of the ischemic myocardium [4]. Those features may explain the improved Left Ventricular Ejection Fraction (LVEF) in patients with Chronic Myocardial Infarction (CMI). It has been shown that selected BMSC were more effective than unselected BMSC in the infarcted myocardium However, the therapeutic efficacy remains controversial. Therefore we conducted a systematic review to examine the effectiveness of selected BMSC on improving LVEF from Randomized Controlled trials (RCTs) to treat CMI.

\section{Methods}

\section{Data search}

We searched Medline from 1946 to March 2012 for studies of BMSC transplantation in patients with CMI. The included studies met the following criteria: randomized controlled trials, CMI patients who received CABG, BMSC were infused intramuscularly, cell injection in peri-infarct zone, and studies that had up to 6 month follow-up to assess the short-term effect of BMSC transplantation.

\section{Data extraction}

Two investigators (HJ and HWY) independently screened all titles and abstracts to identify studies that met the inclusion criteria and extracted relevant data, with divergences resolved by consensus.

*Corresponding author: Hyeon Woo Yim, MD, PhD, Professor, Department of Preventive Medicine, The Catholic University of Korea College of Medicine, 505 Banpo-dong, Seocho-gu, Seoul, Republic of Korea, Tel: +82-2-2258-7860; Fax: +82-2-2258-3820; E-mail: y1693@catholic.ac.kr

Received December 26, 2013; Accepted January 23, 2014; Published January 25, 2014

Citation: Jeong H, Yim HW, Park HJ, Jeong S, Kim H (2014) Are Selected Bone Marrow Stem Cells More Effective than Unselected Ones in Patients with Chronic Myocardial Infarction? J Stem Cell Res Ther 4: 160. doi:10.4172/21577633.1000160

Copyright: ( 2014 Jeong $\mathrm{H}$, et al. This is an open-access article distributed under the terms of the Creative Commons Attribution License, which permits unrestricted use, distribution, and reproduction in any medium, provided the original author and source are credited. 
The details extracted were the study and patient population numbers and characteristics, the type of BMSC, cell dose, baseline LVEF, tools of outcome assessment, whether or not sham injection in the control group, and surgical methods. The outcome data extracted the changes in LVEF from the baseline to 6-month follow-up measured by echocardiography, Magnetic Resonance Imaging (MRI), Single Photon Emission Computed Tomography (SPECT), or left ventricular angiography. Clinical trials with multiple publications and sequential follow-up durations or different outcomes were considered to be one study.

\section{Quality assessment}

Two authors (HJ and HWY) independently assessed the risk of bias for each included study using criteria based on the Cochrane Handbook for Systematic Reviews of Interventions, the principle components of which are sequence generation, allocation concealment, blinding, incomplete outcome data, and selective reporting bias [5]. Disagreements were resolved by discussion between the two authors. Assessment of methodological quality in terms of blindness was assessed by whether or not the control group patients were infused with placebo such as serum or plasma.

\section{Statistical analysis}

All statistics in this meta-analysis were performed using Review Manager Version 5.1 (Copenhagen: The Nordic Cochrane Centre, The Cochrane Collaboration, 2011) [5]. To calculate overall treatment effects weighted mean differences and 95\% confidence intervals were presented. Data were pooled by use of the DerSimonian-Laird randomeffects model due to the high degree of heterogeneity [6]. Heterogeneity was analyzed with the $I^{2}$ statistic, and heterogeneity was defined as low (25-50\%), moderate $(50-75 \%)$, or high $(>75 \%)$. For studies that did not report the actual change from baseline to 6-month follow-up, the change in Standard Deviation (SD) was calculated with a standardized formula used to calculate changes in mean and standard deviation.

\section{Results}

The initial search identified 8,433 references, of which 7 RCTs were, met the inclusion criteria (Figure 1).

The characteristics of the included studies are summarized in Table 1. Three of 7 trials injected selected bone marrow stem cell such as, CD34+ and CD133+ cells $[3,7,8]$ and 4 of 7 trials injected unselected BMSC to the treatment group [9-12]. The treatment effects of the studies in which the treatment group was injected with CD34+ and CD133+ cells (LVEF change of 7.66\%, 95\% CI: 4.16-11.15) were greater than the studies that used unselected BMSC (LVEF change of $4.77 \%$, 95\% CI: 2.08-7.46) (Figure 2).

Table 2 described the methodological quality of RCTs.

\section{Subgroup analyses and investigating heterogeneity}

Subgroup analyses were carried out to assess the impact of the methods used on outcome measure-MRI or echocardiography, the treatment blindness-whether or not the control group patients were infused with placebo such as serum or plasma, and the methods of surgery-conventional CABG or off-pump CABG. Three trials were measured by MRI [9-11] and the other four were measured by echocardiography to assess the LVEF $[3,7,8,12]$. LVEF changes indicated that the treatment effects of the studies that used echocardiography as the outcome measure were greater than those

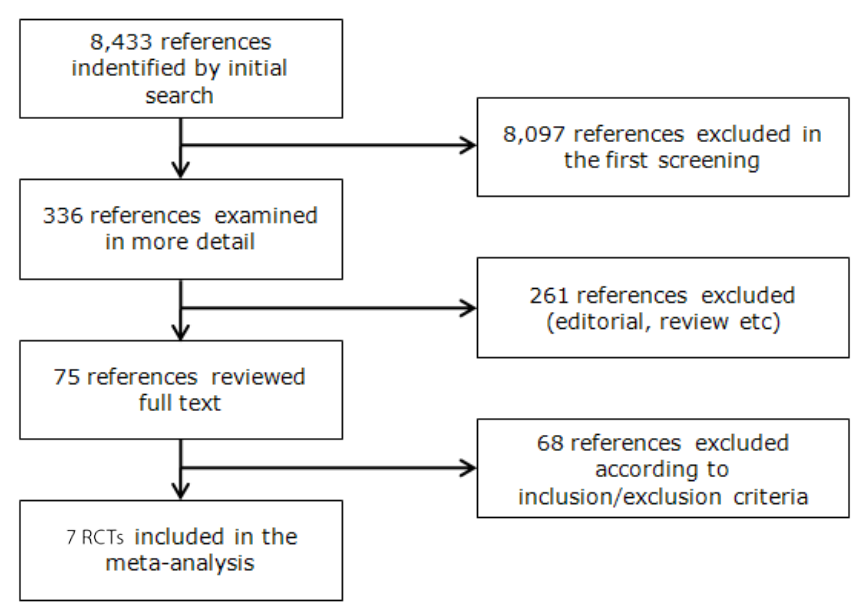

Are-selected-bone-marrow-stem-cells-more-effective-unselected-2157-7633.1000160.pdf

Figure 1: Flow diagram of studies selected in this review.

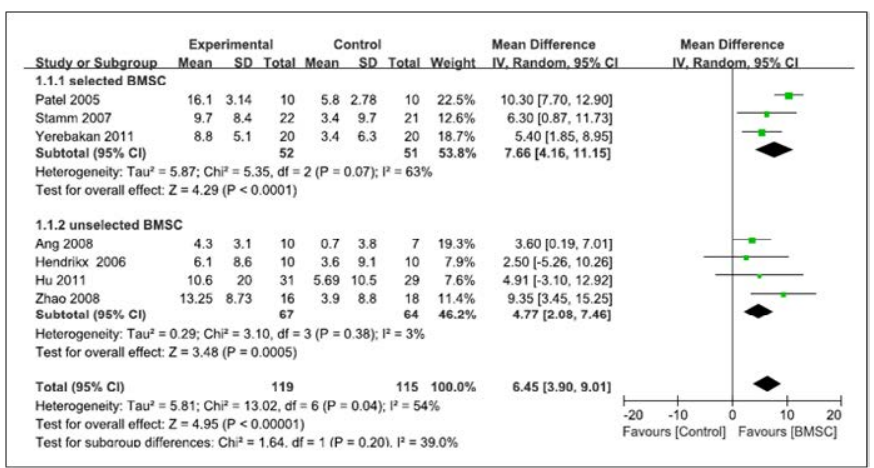

Figure 2: Forest plot of between selected and unselected cell types.

of the studies that used MRI [WMDs: $8.02 \%$ (95\% CI: 5.28-10.77) vs. $3.62 \%$ (95\% CI, 0.71-6.53)]. No statistical heterogeneity was found in the MRI analysis $\left(I^{2}=0 \%\right)$ and moderate statistical heterogeneity was observed in echocardiography $\left(I^{2}=45 \%\right)$.

We also looked at whether or not control group patients were infused with placebo to ensure health care providers remained blind to the study conditions. Three of the 7 trials were infused placebo injection [10-12] and the four remaining trials were not infused with anything in the control group [3,7-9]. The trials without placebo in the control group showed more improvements in LVEF by $8.59 \%$ (95\% CI: 6.49-10.69) than those infused serum (LVEF change of 6.35\%, 95\% CI: 2.29-10.40). A low degree of statistical heterogeneity was found among the placebo injection studies $\left(I^{2}=3 \%\right)$. However, there was substantial heterogeneity in the studies without placebo injection in the control group $\left(I^{2}=79 \%\right)$.

Among the 7 trials, 6 of them used conventional CABG in stem cell transplantation [3,9-12] and only 1 trial used off-pump CABG $(\mathrm{OPCAB})$ [7]. When a subgroup analysis was conducted using only the 5 conventional CABG studies without the $1 \mathrm{OPCAB}$, the heterogeneity disappeared $\left(I^{2}=0 \%\right)$ (Table 3 ). In addition, sensitivity analyses were conducted to estimate the effect of randomization and allocation concealment on LVEF. However, the discrepancies were negligible. 


\begin{tabular}{|c|c|c|c|c|c|c|c|c|c|}
\hline Author & Year & $T(n)$ & $C(n)$ & Cell type & Cell dose $(x 100,000)$ & Baseline EF & Comparator arm & outcome assessment & surgical methods \\
\hline Ang & 2008 & 21 & 21 & unselected BMSC & 84 & 23.2 & No sham injection & MRI & CABG \\
\hline Hendrikx & 2006 & 10 & 10 & unselected BMSC & 60 & 41.2 & Heparinized saline & MRI & CABG \\
\hline $\mathrm{Hu}$ & 2011 & 31 & 29 & unselected BMSC & 131 & 23.7 & patient own serum & MRI & CABG \\
\hline Patel & 2005 & 10 & 10 & selected BMSC & 22 & 30.1 & No sham injection & Echocardiography & OPCAB \\
\hline Stamm & 2007 & 22 & 21 & selected BMSC & 5.8 & 37.7 & No sham injection & Echocardiography & CABG \\
\hline Yerebakan & 2011 & 20 & 20 & selected BMSC & 7.2 & 40.8 & No sham injection & Echocardiography & CABG \\
\hline Zhao & 2008 & 18 & 18 & unselected BMSC & 659 & 36.3 & Heparinized saline & Echocardiography & CABG \\
\hline
\end{tabular}

T (n): Number of Treatment Group Patients; C (n): Number of Control Group Patients; EF: Ejection Fraction; BMSC: Bone Marrow Stem Cell; CABG: Coronary Artery Bypass Graft; MRI: Magnetic Resonance Imaging; OPCAB: Off-Pump Coronary Artery Bypass Grafting

Table 1: Characteristics of included studies.

\begin{tabular}{|c|c|c|c|c|c|}
\hline Author & Year & Randomization & Allocation concealment & Blinding of outcome assessors \\
\hline Ang & 2008 & unclear & unclear & Yes \\
\hline Hendrikx & 2006 & adequate & unclear & Yes \\
\hline Hu & 2011 & adequate & adequate & Yes \\
\hline Patel & 2005 & adequate & adequate & Yes \\
\hline Stamm & 2007 & adequate & unclear & Yes \\
\hline Yerebakan & 2011 & adequate & adequate & Yes \\
\hline Zhao & 2008 & adequate & unclear & 0 \\
\hline
\end{tabular}

Table 2: Risk of bias assessment of included studies.

\begin{tabular}{|c|c|c|}
\hline \multicolumn{3}{|c|}{ Standardized Mean Difference (95\% Confidence interval) $(\%)$} \\
\hline & \multicolumn{2}{|c|}{ Outcome measurement } \\
\hline & MRI & Echocardiography \\
\hline LVEF & $3.62(0.71-6.53)$ & $8.02(5.28-10.77)$ \\
\hline \multirow[t]{3}{*}{ Heterogeneity } & $l^{2}=0 \%$ & $l^{2}=45 \%$ \\
\hline & \multicolumn{2}{|c|}{ Sham injection in the control group } \\
\hline & Yes & No \\
\hline LVEF & $6.35(2.29-10.40)$ & $8.59(6.49-10.69)$ \\
\hline \multirow[t]{3}{*}{ Heterogeneity } & $I^{2}=3 \%$ & $l^{2}=79 \%$ \\
\hline & \multicolumn{2}{|c|}{ Surgical methods } \\
\hline & CABG & OPCAB \\
\hline LVEF & $4.97(2.87-7.07)$ & $10.30(7.7-12.90)$ \\
\hline Heterogeneity & $I^{2}=0 \%$ & not applicable \\
\hline
\end{tabular}

LVEF: Left Ventricular Ejection Fraction; MRI: Magnetic Resonance Imaging; CABG: Coronary Artery Bypass Graft; OPCAB: Off-Pump Coronary Artery Bypass Grafting

Table 3: Subgroup analyses examining the impact outcome measurement, blindness, and surgical methods.

\section{Discussion}

We wanted to directly compare the differences between the selected versus unselected stem cells in the stem-cell treatments for CMI patients. Selected BMSC appeared to show more effective than unselected BMSC (WMDs in LVEF $7.66 \%$ vs. 4.77\%). Our results suggested a potential improvement of heart function after intramyocardial BMSC transplantation for CMI patients, which was indicated by improved LVEF. These findings are consistent with the animal studies. The hematopoietic stem cells and endothelial progenitor cells have been described as cells expressing the hematopoietic marker $\mathrm{CD} 34+$ on their surface. Those cells have the capacity to incorporate in sites of neovascularization and to be differentiated into the endothelial cells in the rat model [13]. In addition, Hofmann et al. [14] have shown that $14 \%$ to $39 \%$ of a CD34-enriched population homed into infarcted myocardium after intracoronary administration, whereas only $1.3 \%$ to $2.6 \%$ of an unselected BMC population did so in patients with acute MI. Those findings suggest that the CD34+ cells may play an important role in successful engraftment of BMC in infarcted cardiac tissue [14].

However, a considerable degree of heterogeneity was observed among the included trials. Our previous finding seemed to indicate that the treatment effects of stem cell therapy in patients with acute MI were associated with design rigorousness explaining the heterogeneity of the effects [15]. Therefore, we wanted to see the treatment effects of stem cell therapy after adjusting for the previously known confounding factors through subgroup analyses according to whether the outcome assessment tool was MRI or echocardiography, whether or not a sham injection was used in the control group, and what type of the surgery method was used.

The assessment of cardiac function is essential for determining the improvement of the myocardium after stem cell transplantation. To measure treatment effect of stem cell therapy on cardiac function for patients with CMI, many researchers used a various aspects of regional myocardial function, such as improvements in global strain and global strain rate, changes in segmental myocardial strain and strain rate, changes in infarct size, regional wall motion, and wall thickening, peak oxygen consumption, 6-minute walk test, and New York Heart Association functional classification. To measure treatment effects of the stem cell therapy on the cardiac function for patients with CMI, various aspects of the regional myocardial function should be considered as well as improvement in the LV systolic and diastolic cardiac function. The diastolic function was influenced by cell necrosis, residual ischemia, microvascular dysfunction, and regional wall motion abnormalities. Exercise capacity is also an important outcome 
measure to assess the myocardial function after MI. Even though those are important functional outcome measures for CMI, they often make it difficult to compare among their effect sizes directly because not all studies used all of them. LVEF has known as a conventional predictor of the myocardial function [16].

The detection of MI in echocardiography showed relatively low sensitivity as well as interpersonal and intrapersonal variability [17]. However, MRI has several important advantages over the other methods for detecting MI, because of its superior spatial resolution and tissue contrast and the unique ability to detect small infarctions that might otherwise be missed in a routine clinical practice using ECG or echocardiography [18]. Because the effects of stem cell therapy are measured by micro-functional improvement in myocardium, a more precise tool may be better for measuring the myocardial function. Moreover, all of the included studies assessed global LVEF as their primary outcome to evaluate the effects of stem cell therapy. Therefore, to increase the comparability we analyzed the changes in global myocardial function to investigate the effect of stem cell transplantation. Global assessment of myocardial function was widely performed by estimating the LVEF using echocardiography or MRI in the included studies. Three trials were measured by MRI and the other four were by echocardiography to assess the LVEF in the present metaanalysis. Up until 6 months of follow-up period there were statistically significant differences LVEF mean change from the baseline measured by both echocardiography and MRI. However, the magnitudes of the improvement were greater with echocardiography than with MRI. This finding was consistent with one of the previous meta-analyses of stem cell treatment for patients with acute myocardial infarction [19].

Clinical trials of stem cell therapy should be strictly designed as it is very difficult to maintain blinding throughout the entire research period, even when research is designed with double-blind controlled trials [20]. We classified the studies into whether or not control group patients were infused with placebo to ensure health care providers remained blind to the study conditions. Three of the 7 trials were infused placebo injection and the four remaining trials were not infused with anything in the control group. The trials without placebo injection in the control group showed more improvements in LVEF than with infused ones. When it comes to the importance of blindness in conducting stem cell transplant to restore the heart function was published recently [15]. Unblinded design might lead to overestimate the treatment effects.

The surgical methods were also one of the important factors of treatment effect. Among the 7 trials, 6 of them used conventional CABG in stem cell transplantation and only 1 trial used OPCAB. The mean changes in LVEF between the baseline and 6 month follow-up were greater OPCAB than conventional CABG.

All of the studies in which the treatment group injected selected CD34+ and CD133+ BMSC assessed the outcome measure with echocardiography and did not infused with anything in the control group. Selected BMSC appeared to be more effective in CMI patients but other factors might have compounded the results. The randomized controlled trial is generally regarded as a strongest study design for assessing benefits or harms of health care interventions. However, randomization in itself does not guarantee that trial results are valid. Methodological issues affecting the validity of randomized controlled trials can occur because all of the data did not come out of doubleblind trials. When it comes to choosing measurement tools, efforts should be made to minimize inter-observer variation which is prone to bias. It is, therefore, not surprising that many studies have found such trials overestimating the treatment effects by a substantial degree when compared with well designed randomized controlled trials. A less rigorous RCT study in which allocation concealments was not kept throughout the study or the measurement tool was not appropriate compared to a well designed study with an objective measurement tool may overestimate the study results. The measurement tools vulnerable to inter-rater variability can also affect the study results. These factors may work as confounders of the study overestimating its effect size.

A systematic review attempts to search all empirical evidence in order to answer a specific research question. The meta-analysis combines results from several different primary studies in order to provide more precise and valid results. In addition to heterogeneity could be explored by conducting subgroup analysis or meta-regression to explain the sources of the heterogeneity since methodological diversity creates heterogeneity through biases variably affecting the results of different studies $[5,21,22]$.

There were some of the limitations. One of the limitations was the small number of studies included in the meta-analysis. Due to the small sample size, we could not conduct a meta-regression to estimate the confounding effects. Second, the changes of LVEF only used for the comparison of myocardial function between selected and unselected stem cells based on the available data.

In conclusion, selected BMSC tended to show more effective than unselected BMSC. The intervention effect of selected BMSC might be overestimated due to the fact that the studies tended to use less rigorous designs, less precise outcome measures, and different methods of surgery than those using unselected BMSC. Therefore these treatment effects of selected cell type should be interpreted cautiously.

\section{Acknowledgements}

This research was supported by a grant (10172MFDS993) from Ministry of Food \& Drug Safety in 2013

\section{References}

1. Beeres SL, Bax JJ, Dibbets-Schneider P, Stokkel MP, Fibbe WE, et al. (2007) Intramyocardial injection of autologous bone marrow mononuclear cells in patients with chronic myocardial infarction and severe left ventricular dysfunction. Am J Cardiol 100: 1094-1098.

2. Li TS, Murakami M, Kobayashi T, Shirasawa B, Mikamo A, et al. (2007) Longterm efficacy and safety of the intramyocardial implantation of autologous bone marrow cells for the treatment of ischemic heart disease. J Thorac Cardiovasc Surg 134: 1347-1349.

3. Stamm C, Kleine HD, Choi YH, Dunkelmann S, Lauffs JA, et al. (2007) Intramyocardial delivery of CD133+ bone marrow cells and coronary artery bypass grafting for chronic ischemic heart disease: safety and efficacy studies. J Thorac Cardiovasc Surg 133: 717-725.

4. Kang HJ, Kim HS, Zhang SY, Park KW, Cho HJ, et al. (2004) Effects of intracoronary infusion of peripheral blood stem-cells mobilised with granulocytecolony stimulating factor on left ventricular systolic function and restenosis after coronary stenting in myocardial infarction: the MAGIC cell randomised clinical trial. Lancet 363: 751-756.

5. Sally Green, Julian PT, Higgins, Philip Alderson, Mike Clarke, et al. (2011) Cochrane Handbook for Systematic Reviews of Interventions. The Cochrane Collaboration, Oxford, UK.

6. DerSimonian R, Laird N (1986) Meta-analysis in clinical trials. Control Clin Trials 7: 177-188.

7. Patel AN, Geffner L, Vina RF, Saslavsky J, Urschel HC Jr, et al. (2005) Surgical treatment for congestive heart failure with autologous adult stem cell transplantation: a prospective randomized study. J Thorac Cardiovasc Surg 130: 1631-1638.

8. Yerebakan C, Kaminski A, Westphal B, Donndorf P, Glass A, et al. (2011) 
Citation: Jeong H, Yim HW, Park HJ, Jeong S, Kim H (2014) Are Selected Bone Marrow Stem Cells More Effective than Unselected Ones in Patients with Chronic Myocardial Infarction? J Stem Cell Res Ther 4: 160. doi:10.4172/2157-7633.1000160

Impact of preoperative left ventricular function and time from infarction on the long-term benefits after intramyocardial CD133(+) bone marrow stem cell transplant. J Thorac Cardiovasc Surg 142: 1530-1539.

9. Ang KL, Chin D, Leyva F, Foley P, Kubal C, et al. (2008) Randomized, controlled trial of intramuscular or intracoronary injection of autologous bone marrow cells into scarred myocardium during CABG versus CABG alone. Nat Clin Pract Cardiovasc Med 5: 663-670.

10. Hendrikx M, Hensen K, Clijsters C, Jongen H, Koninckx R, et al. (2006) Recovery of regional but not global contractile function by the direct intramyocardial autologous bone marrow transplantation: results from a randomized controlled clinical trial. Circulation 114: I101-107.

11. Hu S, Liu S, Zheng Z, Yuan X, Li L, et al. (2011) Isolated coronary artery bypass graft combined with bone marrow mononuclear cells delivered through a graft vessel for patients with previous myocardial infarction and chronic heart failure: a single-center, randomized, double-blind, placebo-controlled clinical trial. J Am Coll Cardiol. 57: 2409-2415.

12. Zhao Q, Sun Y, Xia L, Chen A, Wang Z (2008) Randomized study of mononuclear bone marrow cell transplantation in patients with coronary surgery. Ann Thorac Surg 86: 1833-1840.

13. Asahara T, Kawamoto A (2004) Endothelial progenitor cells for postnatal vasculogenesis. Am J Physiol Cell Physiol 287: C572-579.

14. Hofmann M, Wollert KC, Meyer GP, Menke A, Arseniev L, et al. (2005) Monitoring of bone marrow cell homing into the infarcted human myocardium. Circulation 111: 2198-2202.
15. Jeong H, Yim HW, Cho Y, Park HJ, Jeong S, et al. (2013) The effect of rigorous study design in the research of autologous bone marrow-derived mononuclear cell transfer in patients with acute myocardial infarction. Stem Cell Res Ther 4: 82

16. Nelson GR, Cohn PF, Gorlin R (1975) Prognosis in medically-treated coronary artery disease: influence of ejection fraction compared to other parameters. Circulation 52: 408-412.

17. Catalano O, Moro G, Cannizzaro G, Mingrone R, Opasich C, et al. (2005) Scar detection by contrast-enhanced magnetic resonance imaging in chronic coronary artery disease: a comparison with nuclear imaging and echocardiography. J Cardiovasc Magn Reson 7: 639-647.

18. Barkhausen J, Hunold P, Waltering KU (2004) MRI in coronary artery disease. Eur Radiol 14: 2155-2162.

19. Clifford D, Fisher S, Brunskill S, Doree C, MathurA, et al. (2012) Stem cell treatment for acute myocardial infarction. Cochrane Database of Systematic Reviews, 2.

20. Pildal J, Hróbjartsson A, Jørgensen KJ, Hilden J, Altman DG, et al. (2007) Impact of allocation concealment on conclusions drawn from meta-analyses of randomized trials. Int J Epidemiol 36: 847-857.

21. Antman EM, Lau J, Kupelnick B, Mosteller F, Chalmers TC (1992) A comparison of results of meta-analyses of randomized control trials and recommendations of clinical experts. Treatments for myocardial infarction. JAMA 268: 240-248.

22. Oxman AD, Guyatt GH (1993) The science of reviewing research. Ann N Y Acad Sci 703: 125-133. 